\title{
Probing the surface oxidation of chemically synthesised gold nanospheres and nanorods
}

\author{
Blake J. Plowman • Nathan Thompson • \\ Anthony P. O'Mullane
}

Published online: 3 May 2014

(C) The Author(s) 2014. This article is published with open access at SpringerLink.com

\begin{abstract}
In this study, the electrochemical behaviour of commercially available gold spheres and rods stabilised by carboxylic acid and cetyl trimethyl ammonium bromide (CTAB) moieties, respectively, are investigated. The cyclic voltammetric behaviour in acidic electrolyte is distinctly different with the nanorods exhibiting unusual oxidative behaviour due to an electrodissolution process. The nanospheres exhibited responses typical of a highly defective surface which significantly impacted on electrocatalytic activity. A repetitive potential cycling cleaning procedure was also investigated which did not improve the activity of the nanorods and resulted in deactivating the gold spheres due to decreasing the level of surface defects.
\end{abstract}

Keywords Gold nanoparticles · Electrocatalysis · Active sites

\section{Introduction}

The electrochemical behaviour of metallic nanoparticles has received significant attention due to their applicability as electrocatalysts for a variety of technologically important reactions, in particular, related to fuel cells and electrochemical sensing [1-7]. There are numerous approaches to creating nanoparticles; however, chemical synthesis is particularly attractive given the high degree of control that can be achieved over their size, shape and monodispersity [8]. Central to this is

B. J. Plowman • N. Thompson • A. P. O'Mullane $(\square)$

School of Applied Sciences, RMIT University, GPO Box 2476V,

Melbourne, VIC 3001, Australia

e-mail: anthony.omullane@qut.edu.au

A. P. O’Mullane

School of Chemistry, Physics and Mechanical Engineering, Queensland University of Technology, GPO Box 2434, Brisbane, QLD 4001, Australia the use of capping agents to ensure that nanoparticles do not agglomerate in solution. The effect of size and shape on electrocatalytic performance of metal nanoparticles has been well documented [9-12]. However, there are other factors that need to be considered when assessing the applicability of an electrocatalyst and that is the presence of defect sites and capping agents. In general, it is agreed that the removal of stabilising species from the surface of nanoparticles is required to ensure access to surface active sites that are responsible for electrocatalytic activity [2]. In many cases, this can be quite involved and may perturb the shape and size of the nanomaterial in question. Electrochemical cleaning routes are often used which employ oxidation of the surface to remove the capping agent $[13,14]$. Indeed, repetitive cycling in acidic electrolyte is often used even for bare gold surfaces and nanoparticles to generate a clean sample $[15,16]$. Given the extensive use of gold nanomaterials in catalysis, electrocatalysis, photocatalysis and sensing and their widespread commercial availability, we, therefore, in this work, explore the effect of electrochemical pretreatment of gold nanospheres and nanorods immobilised on a glassy carbon support electrode that are capped with carboxylic acid and cetyl trimethyl ammonium bromide (CTAB) via repetitive potential cycling. Significantly, cyclic voltammetric characterisation in supporting electrolyte alone gives important insights in relation to their oxidation potential as well as their behaviour as electrocatalysts for reactions such as $\mathrm{H}_{2} \mathrm{O}_{2}$ oxidation and reduction and hydrazine oxidation.

\section{Experimental}

Gold nanoparticles stabilised with carboxylic acid ( $10 \pm 2$ and $25 \pm 2 \mathrm{~nm}$ diameter and monodispersity $<2 \%$ coefficient of variation $(\mathrm{CV}))$ and gold nanorods stabilised with $\mathrm{CTAB}$ $(10 \times 50 \mathrm{~nm}$ and $25 \times 256 \mathrm{~nm}, 95 \%$ rods and $<10 \% \mathrm{CV})$ as 
quoted by the manufacturer were purchased from Nanopartz ${ }^{\mathrm{TM}}$ Accurate $^{\mathrm{TM}}$. Aqueous $1 \mathrm{M} \mathrm{H}_{2} \mathrm{SO}_{4}$ (Merck) solutions were made with deionised water (resistivity 18.2 $\mathrm{M} \Omega \mathrm{cm}$ ) purified using a Milli-Q reagent deioniser (Millipore). Hydrazine (BDH), hydrogen peroxide $30 \%(w /$ w) (Sigma) and $\mathrm{KAuBr}_{4}$ (Sigma) were used as received.

Electrochemical experiments were conducted with a $\mathrm{CH}$ Instruments (CHI760C) potentiostat at $20 \pm 2{ }^{\circ} \mathrm{C}$. A glassy carbon (GC) plate electrode $\left(0.158 \mathrm{~cm}^{2} \mathrm{HTW}\right)$ was used as the supporting working electrode and polished using an aqueous $0.3-\mu \mathrm{m}$ alumina slurry, sonicated in deionised water, and dried with a flow of nitrogen gas. Gold nanomaterials with an equivalent optical density of 1 were dropcast $(5 \mu \mathrm{L}$ of $10 \mathrm{~nm}$ $(9 \mathrm{nM}), 25 \mathrm{~nm}(0.5 \mathrm{nM}), 10 \times 50 \mathrm{~nm}(0.9 \mathrm{nM}), 25 \times 256 \mathrm{~nm}$ $(0.04 \mathrm{nM}))$ on the GC electrode and allowed to air dry. The bulk gold electrode (BAS) was $1.6 \mathrm{~mm}$ in diameter and polished using an aqueous $0.3-\mu \mathrm{m}$ alumina slurry, sonicated in deionised water, and dried with a flow of nitrogen gas. The reference electrode was $\mathrm{Ag} / \mathrm{AgCl}$ (aqueous $3 \mathrm{M} \mathrm{KCl}$ ), and the counter electrode was a platinum coil. All electrochemical experiments were commenced after degassing solutions with nitrogen for $10 \mathrm{~min}$.

TEM measurements were performed with a JEOL 1010 TEM operated at an accelerating voltage of $100 \mathrm{kV}$ after dropcasting the nanomaterials on to a carbon-coated TEM grid.

\section{Results and discussion}

The electrochemistry of gold has been widely studied and is often regarded as a model system consisting of an extended double layer region and a monolayer oxide formation/ reduction region as shown in Fig. 1. However, numerous studies have shown that gold is not as inert as its $d^{10}$ configuration suggests which accounts for its pronounced catalytic and electrocatalytic activity [6,17-24]. This has been attributed to active sites on the surface that consists of atoms or clusters of atoms that have low co-ordination number and have the ability to partake in electrocatalytic reactions [21, $22,25,26]$. Recent work by Scholz has demonstrated in the case of gold that active or defect sites are located on the asperities of an electrode surface which are the loci of partially filled $d$ orbitals that can stabilise free radical intermediates [27, 28]. These sites have been shown to dictate electrocatalytic reaction rates for inner sphere reactions that involve free radical intermediates such as oxygen reduction and hydrogen evolution. This was concluded via treatment of gold with Fenton's reagent which dissolved surface asperities and knocked out these active sites as evidenced by a decrease in activity for the reactions mentioned. These active sites were also shown by the same group to be highly important for electrodeposition processes. In a study on platinum electrodeposition on gold electrodes, it was demonstrated that gold electrodes treated with $\mathrm{OH}^{*}$ radicals resulted in a reduction in the number of active sites for Pt nucleation [29]. Prior to this work, Kolb investigated the cyclic voltametric behaviour of single crystal gold surfaces where it was found that there was a significant peak prior to the monolayer oxide formation process that could be attributed to the level of surface defects which were step edges on $\mathrm{Au}(111)$ electrodes [30, 31]. This peak was labelled as OA1 which can also be seen for the bulk polycrystalline electrode shown in Fig. 1. Burke et al. have shown that this peak can be enhanced, as well as other processes well within the double layer region, by thermal and electrochemical activation of bulk gold electrodes [32-34]. This creates a disordered surface which is prone to oxidation at potentials below that for monolayer oxide formation.

To investigate if this type of cyclic voltammetric behaviour is observed at gold nanomaterials, commercially available gold spheres and rods were utilised (Fig. 2). The spheres are capped with carboxylic acid and the rods with cetyl trimethyl ammonium bromide (CTAB). The surfactants were used to prevent aggregation and in the latter case, provide directional growth. The size and shape of these nanomaterials were confirmed by TEM imaging (insets of Fig. 2). It is immediately apparent that the cyclic voltammetric responses for the spheres and rods are significantly different to each other as well as that for a bulk gold electrode (Fig. 1). A distinct OA1 peak occurs prior to monolayer oxide formation on the spherical gold nanoparticles of 10-nm diameter (Fig. 2a). The OA1 process is even more pronounced at the 25 -nm-diameter nanoparticles (Fig. 2c) in relation to the charge passed during oxide formation. As discussed, this peak has been attributed to the presence of surface defect sites when single crystal electrodes were studied but was recently confirmed by Compton [35] at electrodeposited gold nanoparticles which were devoid of capping agents. The oxidation product is speculative in nature but Burke has attributed it to the formation of hydrous oxide type species [26]. Later, Bard demonstrated that incipient oxides are formed on well-polished bulk gold surfaces

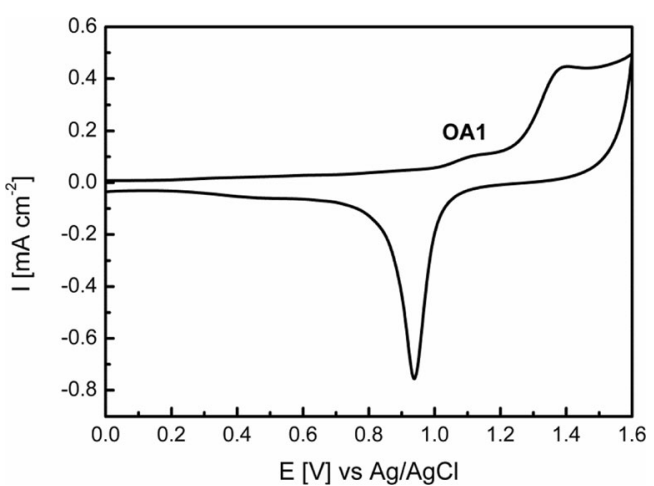

Fig. 1 Cyclic voltammogram recorded in $1 \mathrm{M} \mathrm{H}_{2} \mathrm{SO}_{4}$ at $100 \mathrm{mV} \mathrm{s}^{-1}$ for a bulk gold electrode $(d=1.6 \mathrm{~mm})$ 

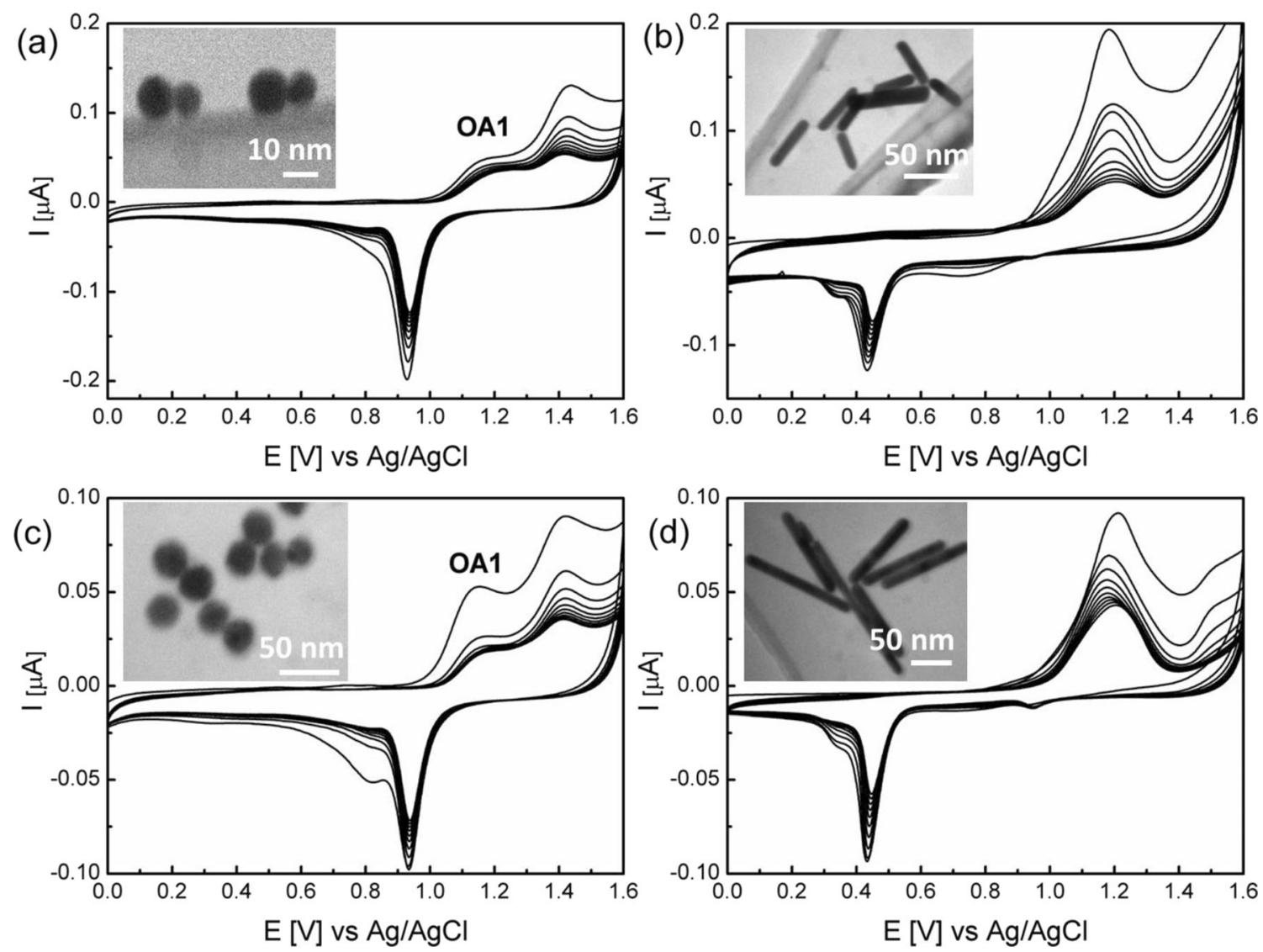

Fig. 2 Cyclic voltammograms recorded in $1 \mathrm{M} \mathrm{H}_{2} \mathrm{SO}_{4}$ at $100 \mathrm{mV} \mathrm{s}^{-1}$ for a 10 -nm Au spheres, b $10 \times 50 \mathrm{~nm} \mathrm{Au}$ rods, c 25 -nm Au spheres and d 25× $256 \mathrm{~nm}$ Au rods. The insets are TEM images of the nanomaterials

with a coverage of 0.2 of a monolayer at potentials within the double layer region [36]. There are several minor features in the double layer region at ca. 0.50 and $0.85 \mathrm{~V}$ for the $10-\mathrm{nm}-$ diameter nanospheres (Fig. 2a) which are more clearly evident from the magnified double layer region shown in Fig. 3. These features have been attributed to the oxidation of adatoms or clusters of adatoms on the surface with low co-ordination number. They were also confirmed to be Faradaic in nature by using large-amplitude Fourier-transformed ac voltammetry and involved in electrocatalytic reactions [25]. These features and the OA1 process are stable upon repetitive cycling for 10 and 25-nm nanoparticles but the overall response does decrease indicating a reduced surface area. However, this demonstrates that the carboxylic acid functional group does not block access to the gold surface.

A dramatic difference in behaviour, however, is observed at the gold nanorods. The onset potential for surface oxidation is similar to the spheres; however, it has a distinctly different shape. It is more reminiscent of a stripping process associated with anodic stripping voltammetry than surface oxide formation which proceeds via $\mathrm{OH}^{-}$ion adsorption followed by a place exchange reaction and further oxidation to a $\mathrm{Au}(\mathrm{III})$ oxide. The major reduction peak is also shifted by a significant amount to ca. $0.42 \mathrm{~V}$ for the nanorods compared to the spheres where the oxide reduction peak occurred at $0.92 \mathrm{~V}$. Therefore, we believe that the reduction peak seen for the nanorods is not related to surface oxide reduction but an entirely different process. CTAB contains $\mathrm{Br}^{-}$, and it is known that gold in the presence of halides oxidises at potentials below that seen for oxide formation [37]. Therefore, the large peak seen on the forward sweep is not due to the OA1 process but rather the electrodissolution of gold. On the reverse sweep, this soluble species which will be present at the electrode surface on the

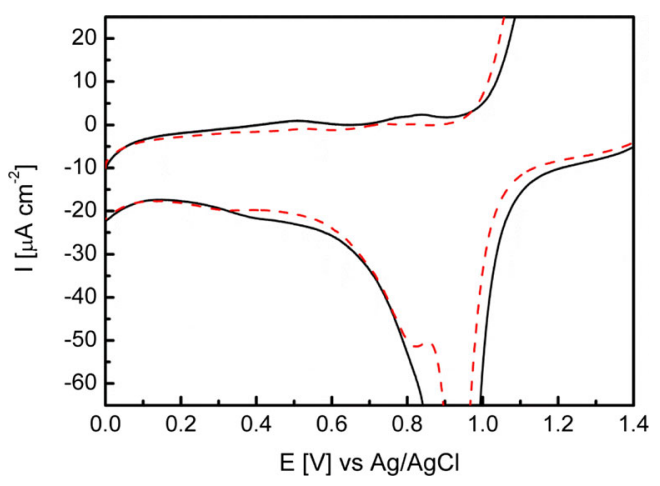

Fig. 3 Cyclic voltammograms recorded in $1 \mathrm{M} \mathrm{H}_{2} \mathrm{SO}_{4}$ at $100 \mathrm{mV} \mathrm{s}^{-1}$ for 10-nm Au spheres (black line) and 25-nm Au spheres (red dash lines) showing the double layer region only 


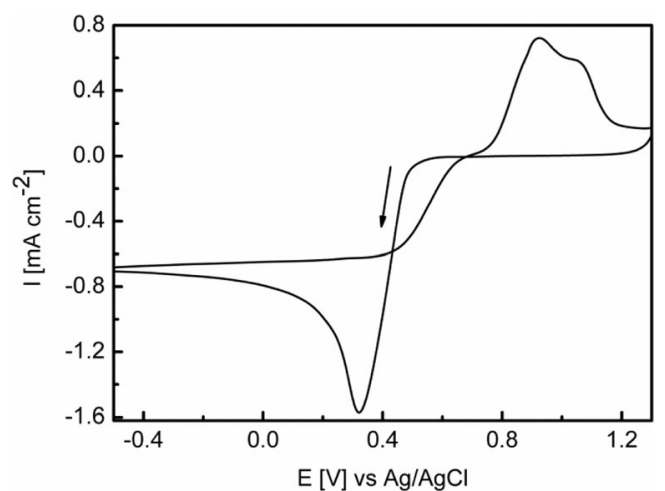

Fig. 4 Cyclic voltammogram obtained at a GC electrode in $10 \mathrm{mM}$ $\mathrm{KAuBr}_{4}$ recorded at $10 \mathrm{mV} \mathrm{s}^{-1}$

timescale of this experiment will be redeposited on the electrode at potentials $<0.60 \mathrm{~V}$. Remarkably, this electrodissolution/re-deposition process is quite stable with only a slight decrease in signal intensity with cycling. The same phenomenon is seen with the higher aspect ratio nanorods, and therefore, the difference in the oxidation onset potential compared to spheres is unlikely to be a size effect. Recent studies have shown that a gold electrode cycled in an aqueous solution of CTAB also undergoes an electrodissolution process where $\mathrm{Au}^{+}$ions are stabilised by bromide in $\mathrm{CTAB}$ which are then redeposited on the reverse sweep at ca. $0.45 \mathrm{~V}$ vs $\mathrm{AgCl}$ [38]. This observation is also consistent with the oxidation of citrate-capped gold nanoparticles in the presence of $\mathrm{KBr}$ which occurred at potentials below that in the absence of bromide in the electrolyte [37]. To investigate this further, gold was electrodeposited from an aqueous solution of $1 \mathrm{mM} \mathrm{KAuBr}_{4}$ on to a GC electrode and is shown in Fig. 4. The peak potential for the deposition of $\mathrm{AuBr}_{4}{ }^{-}$to $\mathrm{Au}^{0}$ is at $0.33 \mathrm{~V}$ which is quite close to that observed in Fig. 2b, d. On the subsequent positive sweep, the electrodissolution of gold can be observed with two peaks at 0.92 and $1.06 \mathrm{~V}$. The overall profile of this response is quite similar to that seen for the oxidation of gold nanorods in $\mathrm{H}_{2} \mathrm{SO}_{4}$ and supports the conclusion that regular monolayer oxide formation does not occur to a significant extent on the CTAB-stabilised nanorods. However, it should be noted that a minor process can be observed on the negative sweep at
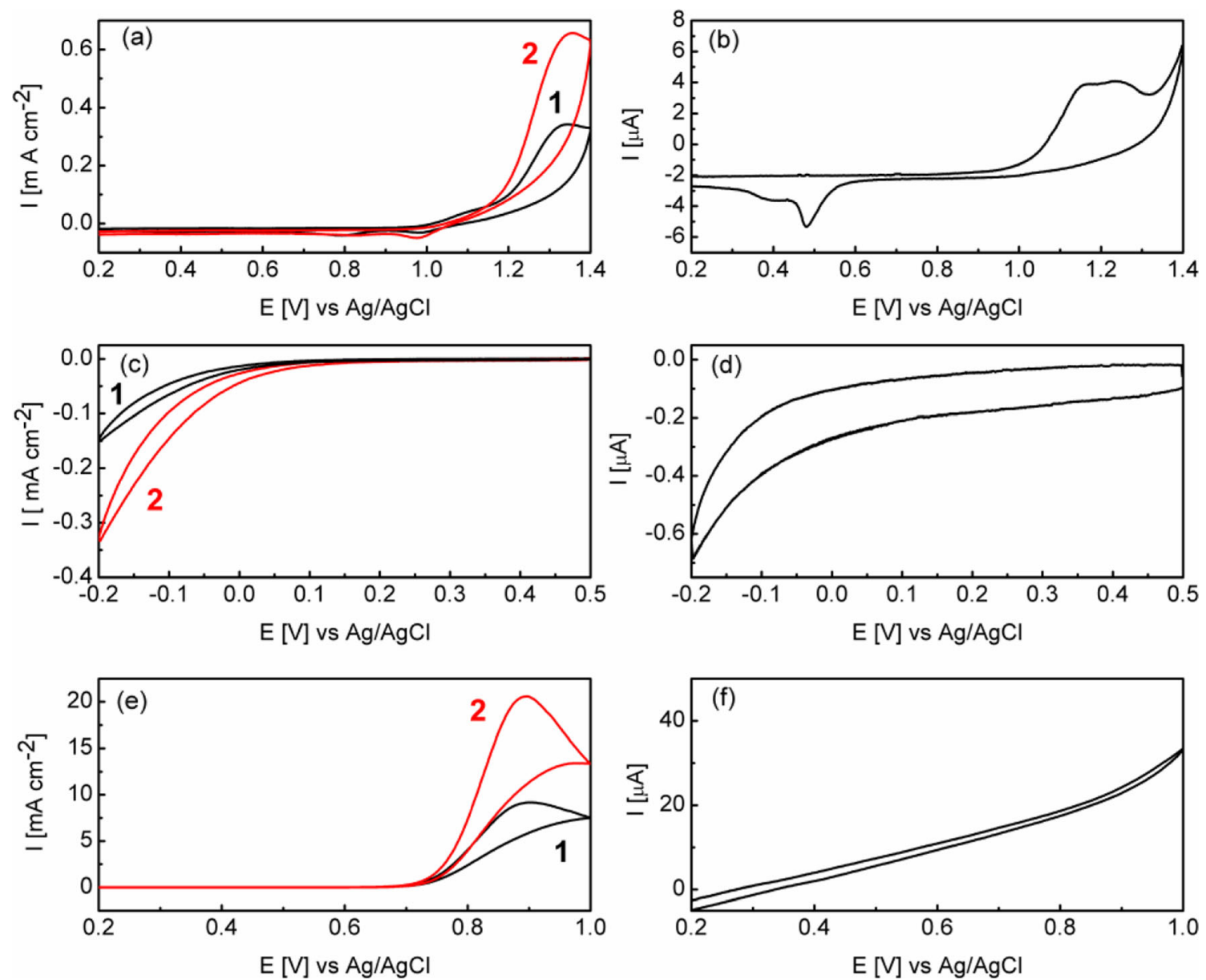

Fig. 5 Cyclic voltammograms in $1 \mathrm{M} \mathrm{H}_{2} \mathrm{SO}_{4}$ containing a-d $20 \mathrm{mM} \mathrm{H}_{2} \mathrm{O}_{2}$ recorded at $20 \mathrm{mV} \mathrm{s}^{-1}$ and $\mathbf{e}-\mathbf{f} 50 \mathrm{mM}$ hydrazine recorded at $50 \mathrm{mV} \mathrm{s}{ }^{-1}$ for 10-nm (1) and 25-nm Au spheres (2). b, d, f Data for $25 \times 256 \mathrm{~nm}$ rods 
Fig. 6 Cyclic voltammograms recorded in $1 \mathrm{M} \mathrm{H}_{2} \mathrm{SO}_{4}$ and $20 \mathrm{mM} \mathrm{H}_{2} \mathrm{O}_{2}$ at $20 \mathrm{mV} \mathrm{s}^{-1}$ at a 25-nm Au spheres and b 25× $256 \mathrm{~nm}$ rods that are pristine (1) and electrochemically cleaned (2)
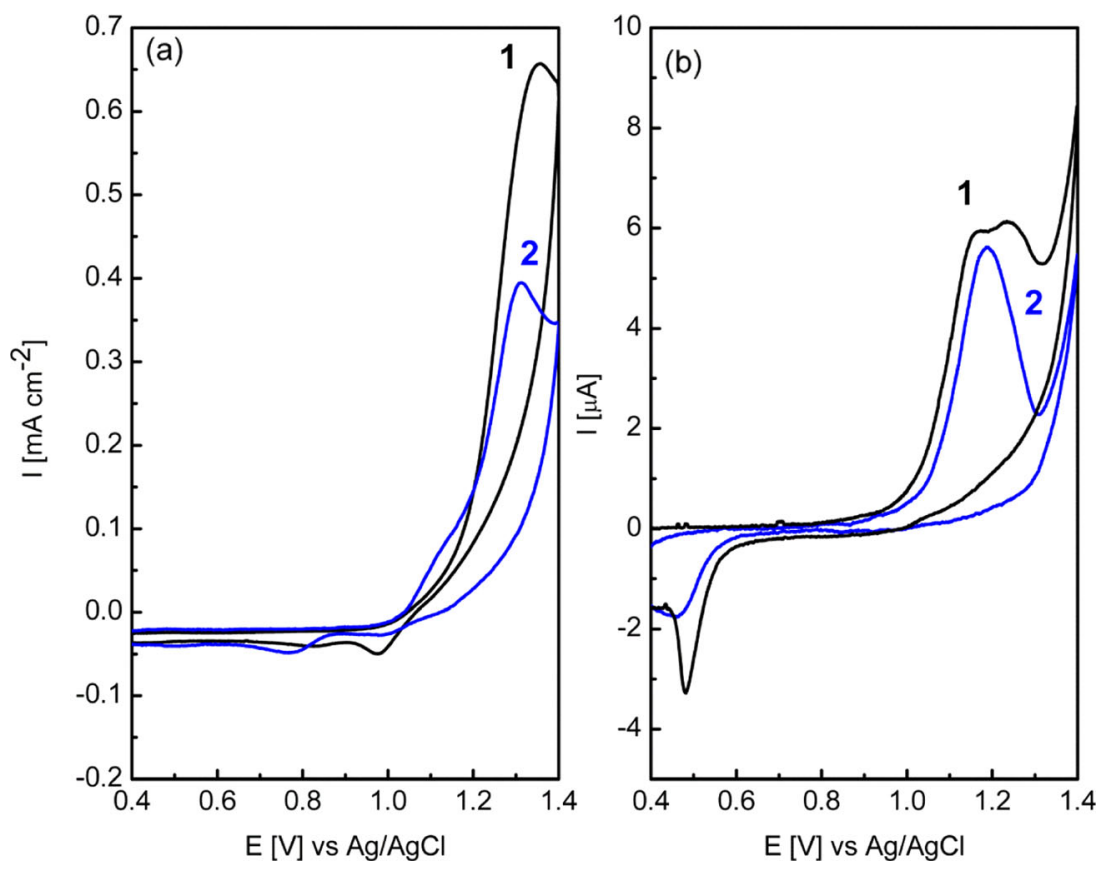

$0.94 \mathrm{~V}$ at the higher aspect ratio nanorods $(25 \times 256 \mathrm{~nm})$ (Fig. 2d) indicating some access to the underlying gold surface.

The oxidation of $\mathrm{H}_{2} \mathrm{O}_{2}$ was chosen to probe the effect of both the capping agent and the role of the OA1 process in electrocatalysis (Fig. 5a, b) where it is clear that activity at the gold nanorods is completely suppressed. A cathodic peak is present at $0.48 \mathrm{~V}$ (Fig. 5b) which suggests that the electrodissolution/re-deposition process described for Fig. 2c still occurs. Koper [39] reported that PVP-stabilised Pt could be cleaned in a solution of $\mathrm{H}_{2} \mathrm{O}_{2} / \mathrm{H}_{2} \mathrm{SO}_{4}$ where $\mathrm{Pt}$ catalyses the decomposition of $\mathrm{H}_{2} \mathrm{O}_{2}$ into water and $\mathrm{O}_{2}$. It was suggested that PVP was physically removed by the generated oxygen bubbles. The electrooxidation of peroxide on gold also generates oxygen; however, in this system, it appears that this is insufficient to remove CTAB from the gold rods. The incipient hydrous oxide adatom mediator (IHOAM) model $[22,40]$ of electrocatalysis predicts that reactions are mediated by a surface-confined redox couple based on the oxidation of active surface sites $\left(\mathrm{M}^{*}\right)$ to hydrous oxide species $(\mathrm{OA} 1$ process). The gold spheres show a clear oxidation process indicating that the carboxylic acid group allows $\mathrm{H}_{2} \mathrm{O}_{2}$ to interact with the surface. In Fig. 5a, current density is presented which indicates the larger nanoparticles have the highest specific activity. This may be related to the intensity of the OA1 process seen in the blank electrolyte and correlates with the IHOAM model which indicates that the formation of a hydrous oxide species at surface defect sites mediates electrocatalytic oxidation reactions. The potential at which this occurs is also consistent with previous studies on electrochemically activated bulk gold electrodes [34].
The electrocatalytic reduction of $\mathrm{H}_{2} \mathrm{O}_{2}$ (Fig. 5c, d) and oxidation of hydrazine (Fig. 5e, f) were also investigated, and the same trend was observed, i.e. activity is completely shut down in the case of the nanorods due to the presence of $\mathrm{CTAB}$ on the surface and that the specific activity of the larger gold spheres was higher. Even though all reactions investigated occur at distinctly different onset potentials, the outcome is the same in that the nanoparticles with the most distinctive OA1 process (in particular on the first sweep) is the most active. This suggests that even though other $M^{*} /$ hydrous oxide transitions are not clearly visible by conventional $\mathrm{dc}$ voltammetry in the double layer region, which require more sophisticated experiments such as large-amplitude Fouriertransformed ac voltammetry to be observed [25], that the $\mathrm{OA} 1$ process may in general be indicative of a highly active surface.

The effect of a repetitive cycling cleaning procedure was then investigated (Fig. 6). The procedure involved cycling between 0.0 and $1.60 \mathrm{~V}$ in $1 \mathrm{M} \mathrm{H}_{2} \mathrm{SO}_{4}$ at $100 \mathrm{mV} \mathrm{s}^{-1}$ for 20 cycles. For the case of the 25 -nm-diameter nanoparticles, there is a decrease in specific activity after the cleaning procedure (note the reduced surface area after cycling is accounted for) which is consistent with the work of Compton who showed a decrease in the activity of electrodeposited gold nanoparticles for the oxygen reduction reaction after a similar procedure [35]. Recent work by Mayrhofer has shown that if a polycrystalline gold electrode is cycled in an acidic solution to potentials where oxide formation occurs, as is the case here, then the electrodissolution of gold occurs [41]. In this study, the lower activity can be correlated with the decrease in intensity of the OA1 process upon repetitive cycling 
(Fig. 2c) as the reduction in surface area via this electrodissolution process has been accounted for. This demonstrates that the density of defect sites which are responsible for the electrooxidation of hydrogen peroxide decreases. The same cleaning procedure was applied to the gold nanorods which showed that this approach is totally ineffective in generating an electrocatalytically active surface (Fig. 6b).

\section{Conclusions}

The electrochemical behaviour of commercially available gold spheres and rods in acidic electrolyte is distinctly different due to the capping agent employed on the surface. The presence of CTAB resulted in an electrodissolution/redeposition process whereas the presence of a carboxylic group allowed surface oxide processes to be observed which indicated the presence of a high level of defect sites. Significantly, a repetitive potential cycling cleaning protocol did not improve the electrocatalytic performance of the nanorods and also led to a slight deactivation of the gold spheres due to a decrease in the number of surface defect sites.

Acknowledgment AOM gratefully acknowledges funding from the Asian Office of Aerospace Research and Development (FA2386-13-1-4073).

Open Access This article is distributed under the terms of the Creative Commons Attribution License which permits any use, distribution, and reproduction in any medium, provided the original author(s) and the source are credited.

\section{References}

1. O'Mullane AP (2014) From single crystal surfaces to single atoms: investigating active sites in electrocatalysis. Nanoscale 6:4012-4026

2. Kleijn SEF, Lai SCS, Koper MTM, Unwin PR (2014) Electrochemistry of nanoparticles. Angew Chem Int Ed 53:3558-3586

3. Campbell CT (2013) The energetics of supported metal nanoparticles: relationships to sintering rates and catalytic activity. Acc Chem Res 46:1712-1719

4. Saha K, Agasti SS, Kim C, Li X, Rotello VM (2012) Gold nanoparticles in chemical and biological sensing. Chem Rev 112:2739-2779

5. Herves P, Perez-Lorenzo M, Liz-Marzan LM, Dzubiella J, Lu Y, Ballauff M (2012) Catalysis by metallic nanoparticles in aqueous solution: model reactions. Chem Soc Rev 41:5577-5587

6. Saint-Lager MC, Laoufi I, Bailly A, Robach O, Garaudee S, Dolle P (2011) Catalytic properties of supported gold nanoparticles: new insights into the size-activity relationship gained from in operando measurements. Faraday Discuss 152:253-265

7. Plowman BJ, Bhargava SK, O'Mullane AP (2011) Electrochemical fabrication of metallic nanostructured electrodes for electroanalytical applications. Analyst 136:5107-5119

8. Sardar R, Funston AM, Mulvaney P, Murray RW (2009) Gold nanoparticles: past, present, and future. Langmuir 25:13840-13851

9. Perez-Alonso FJ, McCarthy DN, Nierhoff A, Hernandez-Fernandez P, Strebel C, Stephens IEL, Nielsen JH, Chorkendorff I (2012) The effect of size on the oxygen electroreduction activity of mass-selected platinum nanoparticles. Angew Chem Int Ed 51:4641-4643

10. Rhee CK, Kim B-J, Ham C, Kim Y-J, Song K, Kwon K (2009) Size effect of Pt nanoparticle on catalytic activity in oxidation of methanol and formic acid: comparison to $\mathrm{Pt}(111), \operatorname{Pt}(100)$, and polycrystalline Pt electrodes. Langmuir 25:7140-7147

11. Chen W, Chen S (2009) Oxygen electroreduction catalyzed by gold nanoclusters: strong core size effects. Angew Chem Int Ed 48:43864389

12. Wang C, Daimon H, Onodera T, Koda T, Sun S (2008) A general approach to the size- and shape-controlled synthesis of platinum nanoparticles and their catalytic reduction of oxygen. Angew Chem Int Ed 47:3588-3591

13. Hebié S, Cornu L, Napporn TW, Rousseau J, Kokoh BK (2013) Insight on the surface structure effect of free gold nanorods on glucose electrooxidation. J Phys Chem C 117:9872-9880

14. El-Deab MS, Sotomura T, Ohsaka T (2005) Oxygen reduction at electrochemically deposited crystallographically oriented $\mathrm{Au}(100)$ like gold nanoparticles. Electrochem Commun 7:29-34

15. Fischer LM, Tenje M, Heiskanen AR, Masuda N, Castillo J, Bentien A, Émneus J, Jakobsen MH, Boisen A (2009) Gold cleaning methods for electrochemical detection applications. Microelectron Eng 86:12821285

16. Carvalhal RF, Sanches Freire R, Kubota LT (2005) Polycrystalline gold electrodes: a comparative study of pretreatment procedures used for cleaning and thiol self-assembly monolayer formation. Electroanalysis 17:1251-1259

17. Shim JH, Kim J, Lee C, Lee Y (2011) Electrocatalytic activity of gold and gold nanoparticles improved by electrochemical pretreatment. J Phys Chem C 115:305-309

18. Lai SCS, Dudin PV, Macpherson JV, Unwin PR (2011) Visualizing zeptomole (electro)catalysis at single nanoparticles within an ensemble. J Am Chem Soc 133:10744-10747

19. Zeng J, Zhang Q, Chen J, Xia Y (2010) A comparison study of the catalytic properties of Au-based nanocages, nanoboxes, and nanoparticles. Nano Lett 10:30-35

20. Zhou X, Xu W, Liu G, Panda D, Chen P (2009) Size-dependent catalytic activity and dynamics of gold nanoparticles at the singlemolecule level. J Am Chem Soc 132:138-146

21. Burke LD (2004) Scope for new applications for gold arising from the electrocatalytic behavior of its metastable surface states. Gold Bull 37:125-135

22. Burke LD, Nugent PF (1998) The electrochemistry of gold. II The electrocatalytic behavior of the metal in aqueous media. Gold Bull 31:39-50

23. Plowman BJ, O'Mullane AP, Bhargava SK (2011) The active site behaviour of electrochemically synthesised gold nanomaterials. Faraday Discuss 152:43-62

24. Plowman BJ, Field MR, Bhargava SK, O’Mullane AP (2013) Exploiting the facile oxidation of evaporated gold films to drive electroless silver deposition for the creation of bimetallic $\mathrm{Au} / \mathrm{Ag}$ surfaces. ChemElectroChem 1:76-82

25. Lertanantawong B, O'Mullane AP, Surareungchai W, Somasundrum M, Burke LD, Bond AM (2008) Study of the underlying electrochemistry of polycrystalline gold electrodes in aqueous solution and electrocatalysis by large amplitude fourier transformed alternating current voltammetry. Langmuir 24:2856-2868

26. Burke LD, Nugent PF (1997) The electrochemistry of gold: I. The redox behavior of the metal in aqueous media. Gold Bull 30:43-53

27. Nowicka AM, Hasse U, Sievers G, Donten M, Stojek Z, Fletcher S, Scholz F (2010) Selective knockout of gold active sites. Angew Chem Int Ed 49:3006-3009

28. Nowicka A, Hasse U, Donten M, Hermes M, Stojek Z, Scholz F (2011) The treatment of $\mathrm{Ag}, \mathrm{Pd}, \mathrm{Au}$ and $\mathrm{Pt}$ electrodes with $\mathrm{OH}^{\circ}$ radicals reveals information on the nature of the electrocatalytic centers. J Solid State Electrochem 15:2141-2147 
29. Sievers G, Hasse U, Scholz F (2012) The effects of pretreatment of polycrystalline gold with $\mathrm{OH}^{*}$ radicals on the electrochemical nucleation and growth of platinum. J Solid State Electrochem 16:1663-1673

30. Schneeweiss MA, Kolb DM, Liu D, Mandler D (1997) Anodic oxidation of $\mathrm{Au}(111)$. Can J Chem 75:1703-1709

31. Kolb DM (2000) Structure studies of metal electrodes by in-situ scanning tunneling microscopy. Electrochim Acta 45:2387-2402

32. Burke LD, Ahern AJ, O'Mullane AP (2002) High energy states of gold and their importance in electrocatalytic processes at surfaces and interfaces. Gold Bull 35:3-10

33. Burke LD, Hurley LM, Lodge VE, Mooney MB (2001) The effect of severe thermal pretreatment on the redox behavior of gold in aqueous acid solution. J Solid State Electrochem 5:250-260

34. Burke LD, O'Mullane AP (2000) Generation of active surface states of gold and the role of such states in electrocatalysis. J Solid State Electrochem 4:285-297

35. Wang Y, Laborda E, Crossley A, Compton RG (2013) Surface oxidation of gold nanoparticles supported on a glassy carbon electrode in sulphuric acid medium: contrasts with the behaviour of 'macro’ gold. Phys Chem Chem Phys 15:3133-3136
36. Rodriguez-Lopez J, Alpuche-Aviles MA, Bard AJ (2008) Interrogation of surfaces for the quantification of adsorbed species on electrodes: oxygen on gold and platinum in neutral Media. J Am Chem Soc 130:16985

37. Masitas RA, Zamborini FP (2012) Oxidation of highly unstable $<4 \mathrm{~nm}$ diameter gold nanoparticles $850 \mathrm{mV}$ negative of the bulk oxidation potential. J Am Chem Soc 134:5014-5017

38. Nambiar SR, Aneesh PK, Rao TP (2014) Studies on deposition of gold atomic clusters on to polycrystalline gold electrode from aqueous cetyl trimethyl ammonium bromide solutions. J Electroanal Chem 722-723:60-67

39. Monzó J, Koper MTM, Rodriguez P (2012) Removing polyvinylpyrrolidone from catalytic Pt nanoparticles without modification of superficial order. ChemPhysChem 13:709-715

40. Burke LD, Casey JK, Morrissey JA, Murphy MM (1991) Incipient hydrous oxide/adatom mediator (IHOAM) model of electrocatalysis. Bull Electrochem 7:506-511

41. Cherevko S, Topalov AA, Katsounaros I, Mayrhofer KJJ (2013) Electrochemical dissolution of gold in acidic medium. Electrochem Commun 28:44-46 